\title{
Conformal freeform surfaces
}

\author{
Yi-Jun Yang a,b,* Wei Zeng ${ }^{c}$ Xiang-Xu Meng ${ }^{\text {a,b }}$ \\ ${ }^{a}$ School of Computer Science \& Technology, Shandong Univ., Jinan, China \\ ${ }^{\mathrm{b}}$ Engineering Research Center of Digital Media Tech., MOE of China \\ ${ }^{\mathrm{c}}$ School of Computing \& Information Sciences, Florida International Univ., USA
}

\begin{abstract}
The conformality of freeform surfaces highly affects their rendering and tessellation results. To improve the conformality of freeform surfaces, a novel freeform surface representation named hierarchical freeform surfaces is presented in this paper. The conformality energy of hierarchical freeform surfaces is first formulated and its numerical approximation is then constructed using the composite Simpson's rule. By constructing the parameterization of the initial freeform transformation using the Ricci flow method, the optimal freeform transformation is obtained by the Levenberg-Marquardt method, which is further interleaved with the freeform refinement procedure to generate a hierarchical freeform surface with bounded conformality deviations. Examples are given to show the performance of our algorithm for rendering and tessellation applications.
\end{abstract}

Key words: Hierarchical freeform surfaces; Conformal surfaces; Least square method

\section{Introduction}

Freeform surfaces play an increasingly important role in contemporary graphics and architecture [29]. The results of freeform related applications, such as surface rendering (e.g. texture mapping), surface tessellation, surface sampling, and so on, are highly dependent on the surface parameterization [3$5,16,23,31,34-36,46,49,52,53]$. A NURBS surface has an intrinsic rational piecewise polynomial mapping from the $3 \mathrm{D}$ surface to the $2 \mathrm{D}$ parameter domain (see Figure 1). By surface reparameterizations [25,49,52,53], the surface may

\footnotetext{
* Corresponding author. Address: No. 1500, Shunhua Road, Gaoxin District, Shan-
} dong University, Jinan, China. Postal Code: 250100. Email address: yyj@sdu.edu.cn.

Preprint submitted to Elsevier 9 August 2016

(C) 2016. This manuscript version is made available under the Elsevier user license

http://www.elsevier.com/open-access/userlicense/1.0/ 


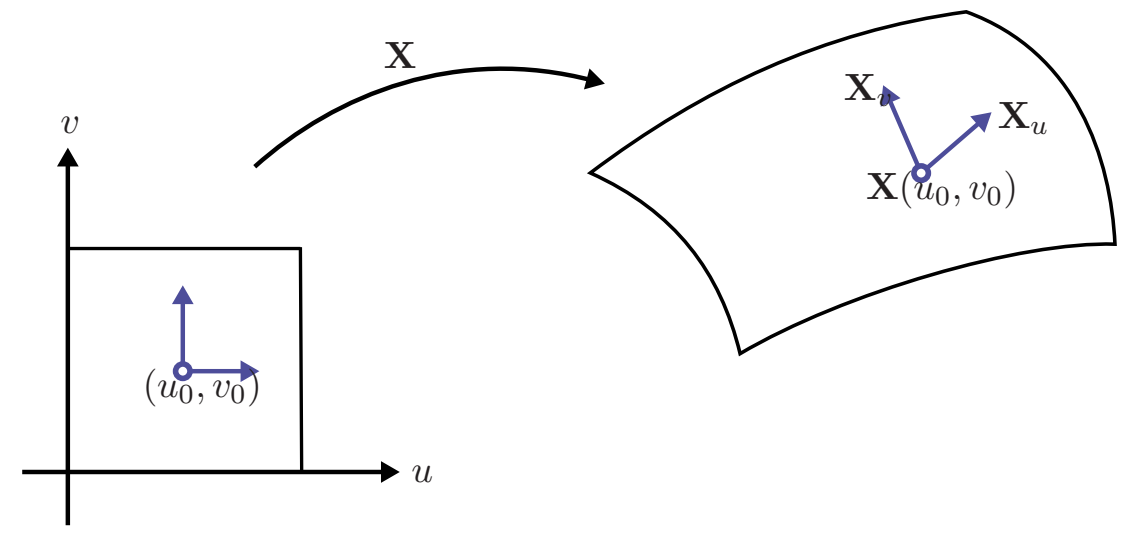

Fig. 1. The intrinsic mapping of a NURBS surface. A $2 \mathrm{D}$ point $\left(u_{0}, v_{0}\right)$ in the parameter domain is mapped to a $3 \mathrm{D}$ point $\mathbf{X}\left(u_{0}, v_{0}\right)$ on the surface. The two partial derivatives $\mathbf{X}_{u}\left(u_{0}, v_{0}\right)$ and $\mathbf{X}_{v}\left(u_{0}, v_{0}\right)$ at the point $\mathbf{X}\left(u_{0}, v_{0}\right)$ may be not orthogonal and have different norm lengths.

have infinitely many different parameterizations. Depending on where and how it will be used, one may need to find a suitable or optimal parameterization out of the infinitely many, or to convert the given parameterization into another (more) suitable one $[25,49,52,53]$. In many applications, such as texture mapping, surface tessellation, surface matching and registration, it is highly desirable that the parameterization is shape preserving (conformal) i.e. maps an elementary circle of the parameter domain to an elementary circle of the surface. At the same time, the surface modifications (changing the surface control points and/or weights) and surface fitting in reverse engineering both may introduce NURBS surfaces with parameterizations far from being conformal.

Research on conformal mappings has been wide and varied [14,37,57]. While most of the successes have been reported in generating the conformal mapping of triangle meshes, conformality of complex freeform surfaces has not met with similar achievements. From our point of view, the lack of conformal parameterizations is the bottleneck for freeform surfaces to achieve high quality results for rendering and tessellation applications. Moveover, a conformal parameterization will lead to more robust and stable computations for derivative based algorithms such as surface sampling, surface intersection, curvature computation, and so on $[3,31,36,49]$.

\subsection{Related works}

We focus on review on freeform surface parameterization methods. For triangle meshes, we concentrate on analyzing the difference between their parameterization and freeform surface parameterization, a detailed review of mesh parameterization techniques being beyond the scope of this article. For the details of mesh parameterization, the reader is recommended to see the survey 
papers $[14,19,39]$ and the references therein.

The parameterization of triangle meshes has been studied extensively in the last decade and still remains as a hot topic until now $[14,15,19,26,30,37,39,57]$. The main purpose of the research on the parameterization of triangle meshes is to construct a suitable, bijective mapping between the triangle mesh embedded in $3 \mathrm{D}$ and a simple 2D domain, referred to as the parameter space or parameter domain. To minimize the parameterization distortion in terms of either angles or areas, many different algorithms have been proposed in the literature $[14,19,30,37,39,57]$. As the NURBS surface already has an intrinsic rational polynomial mapping (see Figure 1) from the 2D parameter domain (a rectangle) to the 3D surface, its parameterization has some specific properties different from the parameterization of triangle meshes. First, the research on conformality of freeform surfaces is more complex than that on the triangle meshes. Only a small number of NURBS surfaces are conformal and a general NURBS surface is not. Second, the NURBS surface has an intrinsic mapping already and we don't need to construct an initial surface mapping from the 3D surface to the $2 \mathrm{D}$ parameter domain any more, which is the case for triangle meshes. Third, the parameterization of NURBS surface is a continuous rational polynomial mapping while those of triangle meshes are discrete, usually defined by the correspondence between their vertices and the correspondence of points inside the triangles is obtained from the vertices correspondence by interpolation techniques. If we convert the NURBS surface into a triangle mesh and apply the mesh parameterization method, some parameterization results can be obtained subsequently. However, there is one main drawback for this kind of methods. The resultant surface representation (discrete triangle representation) is not continuous anymore, which is problematic for subsequent algorithms designed for freeform surfaces. Though we can reconstruct the freeform surface by traditional least-square fitting methods from the triangle parameterization, neither the surface shape nor the triangle parameterization are preserved precisely during the fitting procedure, which is not allowed for high accurate freeform related applications.

Recent GPU advances have given rise to the possibility of using freeform surfaces for real-time applications. For example, DirectX 11 adds hardware support for tessellation of arbitrary parametric surfaces. Yet the quality of the tessellation highly depends on its parameterization. In this paper, we give a novel representation of freeform surfaces named hierarchical freeform surfaces, which enjoys the hardware support for tessellation as the transformation itself is represented as a 2D freeform surface also. The only difference is to perform the tessellation procedure twice, the first for the transformation surface and the second for the 3D parametric surface.

The aim of this paper is to optimize the conformality of given freeform surfaces for applications such as surface visualization, surface sampling, surface 


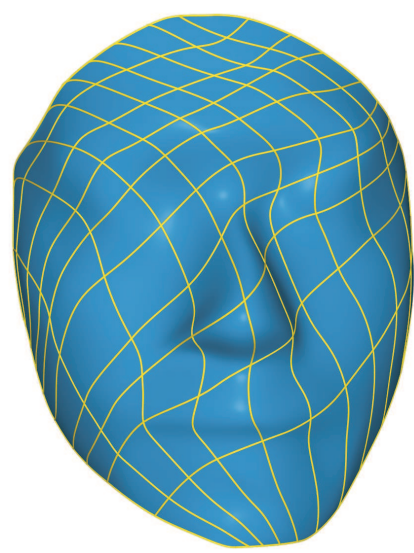

(a) face surface

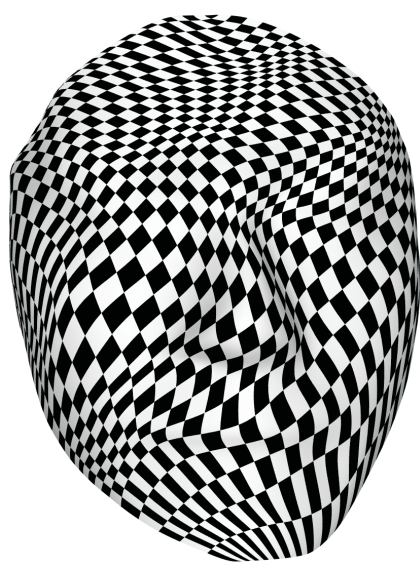

(b) texture mapping

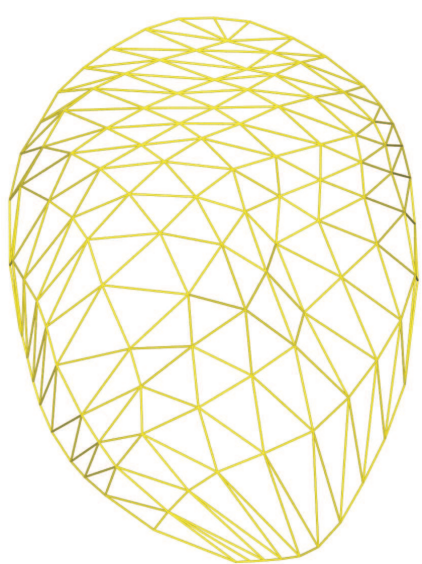

(c) tessellation result

Fig. 2. Texture mapping and tessellation of the face surface. (a) the face parameterization; (b) the texture mapping of a checkerboard image on the surface; (c) the tessellation of mapping a uniform triangle subdivision of the parameter domain onto the surface.

tessellation, surface intersection, curvature computation and so on. Most of all, we want to keep the surface geometry (the surface shape) unchanged and let the resultant surfaces represented as continuous representations, which is preferable and convenient for the algorithms designed for the above mentioned applications. The method presented in this paper utilizes the freeform transformation, which satisfies both the surface shape and representation requirements.

Compared with the quantitative study of the triangle mesh parameterization $[6,13,14,17,18,21,33,37,41,43-45,47,48,56,57]$, little attention has been paid to the freeform surface parameterization until now. The results of rendering and tessellation applications for NURBS surfaces largely depend on the parameterization quality. To perform texture mappings on a NURBS surface, the parametric coordinate of the surface is usually reused as the texture coordinate. If the parameterization is far from being conformal, there will be large distortion of the texture image on the surface (see Figure 2(b)). To tessellate a NURBS surface, most existing algorithms [16,31,34] map a triangulation of the parameter domain onto the surface. Similar to texture mapping, the final tessellation results largely depend on the surface parameterization (see Figure $2(\mathrm{c})$ ). A conformal surface parameterization not only preserves the appearance of texture, but also avoids degenerate elements for the tessellation application. Moreover, a conformal parameterization will lead to more robust and stable computations for derivative based algorithms such as surface sampling, surface intersection, curvature computation, and so on $[3,31,36,49]$. He et al. [18] gave a rational bicubic reparameterization method to improve the parameterization of the approximate Gregory patches such that the new parameterization conforms better to that of the given subdivision surface. Both the explicit rep- 
resentation of the reparameterized surface and the conformality of the final surface are not considered therein. Yang et al. [50] presented an algorithm to improve the Bézier surface parameterization based on Möbius transformations $[10,50]$, which can change only the distribution of iso-parametric curves, but not the shape of them. To obtain more uniform iso-parametric curves, a rational bilinear reparameterization algorithm was also presented in [50]. However, only the uniformity of iso-parametric curves was considered. Furthermore, the rational bilinear reparameterization coefficients are determined by a trivial interpolation method, which is only suitable for a special surface case. To obtain more uniform and orthogonal iso-parameter curves for rational Bézier surfaces, Yang et al. [54] presented an optimization algorithm to minimize the nonlinear energy measuring uniformity and orthogonality deviations using the rational bilinear reparameterizations, which produces a better parameterization with the cost of degree elevation. To further improve the conformality of freeform surfaces, Yang et al. [55] presented another optimization algorithm using the general bilinear transformations. The initial general bilinear transformation is obtained by approximating the conformal mapping of its 3D discretized mesh using a least square method, which is then optimized by the LevenbergMarquardt method. However, both the Möbius transformation and bilinear transformations presented in $[50,54,55]$ don't have sufficient ability to obtain satisfying conformal parameterizations with bounded conformal deviations.

\subsection{Algorithm Overview}

A novel representation of freeform surfaces named hierarchical freeform surfaces is presented in this paper. Based on the hierarchical freeform surface, we present an optimization algorithm to improve the conformality of given freeform surfaces. In the optimization procedure, a nonlinear energy measuring the conformality deviations is first formulated and its discrete version is then constructed using the composite Simpson's rule. By constructing the parameterization of the initial freeform transformation using the Ricci flow method, the optimal freeform transformation is obtained by a nonlinear least square method, which is further interleaved with the freeform refinement procedure to generate a hierarchical freeform surface with bounded conformality deviations. Though we utilize NURBS surfaces as the freeform representation in this paper, our method can be generalized to other freeform representations such as T-spline and Gregory surfaces with little modifications. Examples are given to show the performance of our algorithm for tessellation and visualization applications.

The paper is organized as follows. Section 2 describes the hierarchical freeform surfaces and their differential properties. In Section 3, we show how to optimize the conformality of given NURBS surface using the freeform transformations. 
Several examples are given to show the performance of our algorithm in Section 4 and Section 5 concludes the paper.

\section{Hierarchical freeform surfaces}

In this paper, we adopt NURBS surfaces as the freeform representation, which can be generalized to other freeform representations with corresponding modifications. For a NURBS surface, its hierarchical freeform representation can be obtained by redefining its parameters $(u, v)$ with a two dimensional transformation $(u, v)=\phi(s, t)$, which is also a freeform surface. The underlying surface geometry is unchanged by defining the hierarchical freeform representation. What changes is the surface parameterization. The basic motivation of introducing hierarchical freeform surfaces is to improve the parameterization of freeform surfaces for the related applications such as surface visualization, surface tessellation, surface sampling, surface blending while keeping their original geometry as well as their parametric continuity. The framework of hierarchical freeform surfaces adapts well to the current GPU architecture by performing the GPU algorithms for traditional freeform surfaces twice. Here we first describe the definition of hierarchical freeform surfaces followed by the analysis of their differential geometry properties.

\subsection{Definition}

A NURBS surface in three dimensional space is defined by

$$
\mathbf{X}(u, v)=\frac{\sum_{i=0}^{n_{u}} \sum_{j=0}^{n_{v}} N_{i}^{p_{1}}(u) N_{j}^{q_{1}}(v) \omega_{i, j} \mathbf{P}_{i, j}}{\sum_{i=0}^{n_{u}} \sum_{j=0}^{n_{v}} N_{i}^{p_{1}}(u) N_{j}^{q_{1}}(v) \omega_{i, j}}, \quad u, v \in[0,1]
$$

where $\mathbf{P}_{i, j}$ are the control points, $\omega_{i, j}$ are the weights, and $N_{i}^{p_{1}}(u), N_{j}^{q_{1}}(v)$ are the $p_{1}$ th-degree and $q_{1}$ th-degree $\mathrm{B}$-spline basis functions defined on the knot vectors

$$
\mathbf{U}=\{\underbrace{u_{p_{1}}, \ldots, u_{p_{1}}}_{p_{1}+1}, u_{p_{1}+1}, \ldots, u_{n_{u}}, \underbrace{u_{n_{u}+1}, \ldots, u_{n_{u}+1}}_{p_{1}+1}\}
$$

and

$$
\mathbf{V}=\{\underbrace{v_{q_{1}}, \ldots, v_{q_{1}}}_{q_{1}+1}, v_{q_{1}+1}, \ldots, v_{n_{v}}, \underbrace{v_{n_{v}+1}, \ldots, v_{n_{v}+1}}_{q_{1}+1}\},
$$


respectively. For the hierarchical freeform representation, $(u, v)$ parameters are subject to the following freeform transformation.

$$
(u, v)=\phi(s, t)=\sum_{i=0}^{n_{s}} \sum_{j=0}^{n_{t}} N_{i}^{p_{2}}(s) N_{j}^{q_{2}}(t) \mathbf{Q}_{i, j},
$$

where $\mathbf{Q}_{i, j}$ are the $2 \mathrm{D}$ control points, and $N_{i}^{p_{2}}(s), N_{j}^{q_{2}}(t)$ are the $p_{2}$ th-degree and $q_{2}$ th-degree B-spline basis functions defined on their knot vectors

$$
\mathbf{S}=\{\underbrace{u_{p_{2}}, \ldots, u_{p_{2}}}_{p_{2}+1}, u_{p_{2}+1}, \ldots, u_{n_{u}}, \underbrace{u_{n_{u}+1}, \ldots, u_{n_{u}+1}}_{p_{2}+1}\},
$$

and

$$
\mathbf{T}=\{\underbrace{v_{q_{2}}, \ldots, v_{q_{2}}}_{q_{2}+1}, v_{q_{2}+1}, \ldots, v_{n_{v}}, \underbrace{v_{n_{v}+1}, \ldots, v_{n_{v}+1}}_{q_{2}+1}\}
$$

respectively. In this paper, we use a $3 \times 3$ degree freeform transformation with uniform knot vectors.

\subsection{Fundamental Algorithms of hierarchical freeform surfaces}

The traditional geometric methods can adapt well with the new representation such as point evaluation, derivative computation, point inversion, surface tangent vector inversion and so on without the explicit representation of the resultant surface. The detailed procedure are described as follows.

1. Point Evaluation. Given a $2 \mathrm{D}$ point $\left(s_{0}, t_{0}\right)$ in the parameter domain, its corresponding point on surface $\mathbf{X}$ can be obtained by first computing the $2 \mathrm{D}(u, v)$ coordinates and substituting them into the original surface parametric equation $\mathbf{X}\left(\phi\left(s_{0}, t_{0}\right)\right)$.

2. Derivative Computation. The derivative of the new representation can be obtained by first computing the derivatives of the NURBS surface and the freeform transformation separately and combining them using the chain rule

$$
\mathbf{X}_{s}=\mathbf{X}_{u} \cdot u_{s}+\mathbf{X}_{v} \cdot v_{s} \text { and } \mathbf{X}_{t}=\mathbf{X}_{u} \cdot u_{t}+\mathbf{X}_{v} \cdot v_{t}
$$

3. Point Inversion. Given a point $\mathbf{P}$, assumed to lie on the NURBS surface $X$, point inversion is the problem of finding the corresponding parameter $\left(s_{0}, t_{0}\right)$, such that $\mathbf{X}\left(\phi\left(s_{0}, t_{0}\right)\right)=\mathbf{P}$. The point inversion of the hierarchical freeform surfaces can be obtained by performing the traditional point inversion method twice, the first for the NURBS surface and the second for the freeform transformation. 
4. Surface Tangent Vector Inversion. Let $\left(s_{0}, t_{0}\right)$ be a fixed parameter point and $\mathbf{P}=\mathbf{X}\left(\phi\left(s_{0}, t_{0}\right)\right)$ its image on the hierarchical freeform surface. Let $\mathbf{T}=(d x, d y, d z)$ be a vector starting at $\mathbf{P}$ and lying in the tangent plane of the hierarchical freeform surface at $\mathbf{P}$. Denote by $\mathbf{X}_{s}$ and $\mathbf{X}_{t}$ the first partial derivatives at $\left(s_{0}, t_{0}\right)$. If $\mathbf{X}_{s} \times \mathbf{X}_{t} \neq 0$, it follows from elementary differential geometry that there exists a vector $\mathbf{W}=(d s, d t)$ in the st plane such that

$$
\mathbf{T}=\mathbf{X}_{s} d s+\mathbf{X}_{t} d t
$$

Tangent vector inversion is the process of determining the vector $\mathbf{W}$. Surface tangent vector inversion of the hierarchical freeform surfaces can be obtained by first computing the derivatives of the hierarchical freeform surfaces, then obtaining the vector $\mathbf{W}=(d s, d t)$ from the following equations

$$
\left\{\begin{array}{l}
\mathbf{T} \cdot \mathbf{X}_{s}=\left(\mathbf{X}_{s} \cdot \mathbf{X}_{s}\right) d s+\left(\mathbf{X}_{t} \cdot \mathbf{X}_{s}\right) d t \\
\mathbf{T} \cdot \mathbf{X}_{t}=\left(\mathbf{X}_{s} \cdot \mathbf{X}_{t}\right) d s+\left(\mathbf{X}_{t} \cdot \mathbf{X}_{t}\right) d t
\end{array}\right.
$$

After we give the fundamental algorithms of hierarchical freeform surfaces, their differential properties are described in the following subsection.

\subsection{Differential Properties}

The parameterization in Equation (1) is characterized by its first fundamental form $[9]$

$$
d s^{2}=\mathbf{X}_{u} \cdot \mathbf{X}_{u}(d u)^{2}+2 \mathbf{X}_{u} \cdot \mathbf{X}_{v} d u d v+\mathbf{X}_{v} \cdot \mathbf{X}_{v}(d v)^{2},
$$

where $\mathbf{X}_{u}=\frac{\partial \mathbf{X}}{\partial u}$ and $\mathbf{X}_{v}=\frac{\partial \mathbf{X}}{\partial v}$ are the two partial derivative vectors of the surface $\mathbf{X}$. The first fundamental form describes the metric on the surface $\mathbf{X}$. Let

$$
E=\mathbf{X}_{u} \cdot \mathbf{X}_{u}, \quad F=\mathbf{X}_{u} \cdot \mathbf{X}_{v}, \quad G=\mathbf{X}_{v} \cdot \mathbf{X}_{v}
$$

and rewrite the coefficients in a symmetric matrix

$$
\mathbf{I}=\left(\begin{array}{ll}
E & F \\
F & G
\end{array}\right)
$$


where $E$ and $G$ give the square length of the two partial derivatives and $F$ measures the orthogonality of the two partial derivatives. Then, we have

$$
d s^{2}=\left(\begin{array}{ll}
d u & d v
\end{array}\right) \mathbf{I}\left(\begin{array}{l}
d u \\
d v
\end{array}\right) .
$$

Using the chain rule, we can obtain the partial derivatives of hierarchical freeform surfaces over the new parametric variables $(s, t)$ as follows

$$
\begin{aligned}
& \mathbf{X}_{s}^{T}=\left(\begin{array}{ll}
\mathbf{X}_{u}^{T} & \mathbf{X}_{v}^{T}
\end{array}\right) \phi_{s}^{T} \\
& \mathbf{X}_{t}^{T}=\left(\begin{array}{ll}
\mathbf{X}_{u}^{T} & \mathbf{X}_{v}^{T}
\end{array}\right) \phi_{t}^{T}
\end{aligned},
$$

where $\phi_{s}$ and $\phi_{t}$ are the partial derivatives of the 2D transformation surface defined in Equation (2) over the variables $s$ and $t$, respectively. The first fundamental terms of the hierarchical freeform surfaces are as follows.

$$
\begin{aligned}
\widetilde{E} & =\mathbf{X}_{s} \cdot \mathbf{X}_{s}=\mathbf{X}_{s} \mathbf{X}_{s}^{T} \\
& =\phi_{s}\left(\begin{array}{l}
\mathbf{X}_{u} \\
\mathbf{X}_{v}
\end{array}\right)\left(\begin{array}{ll}
\mathbf{X}_{u}^{T} & \mathbf{X}_{v}^{T}
\end{array}\right) \phi_{s}^{T} \\
& =\phi_{s}\left(\begin{array}{cc}
E & F \\
F & G
\end{array}\right) \phi_{s}^{T} \\
& =\phi_{s} \mathbf{I} \phi_{s}^{T}, \\
\widetilde{F} & =\mathbf{X}_{s} \cdot \mathbf{X}_{t}=\mathbf{X}_{s} \mathbf{X}_{t}^{T} \\
& =\phi_{s}\left(\begin{array}{l}
\mathbf{X}_{u} \\
\mathbf{X}_{v}
\end{array}\right)\left(\begin{array}{ll}
\mathbf{X}_{u}^{T} & \mathbf{X}_{v}^{T}
\end{array}\right) \phi_{t}^{T} \\
& =\phi_{s} \mathbf{I} \phi_{t}^{T},
\end{aligned}
$$

and

$$
\begin{aligned}
\widetilde{G} & =\mathbf{X}_{t} \cdot \mathbf{X}_{t}=\mathbf{X}_{t} \mathbf{X}_{t}^{T} \\
& =\phi_{t}\left(\begin{array}{l}
\mathbf{X}_{u} \\
\mathbf{X}_{v}
\end{array}\right)\left(\begin{array}{ll}
\mathbf{X}_{u}^{T} & \mathbf{X}_{v}^{T}
\end{array}\right) \phi_{t}^{T} \\
& =\phi_{t} \mathbf{I} \phi_{t}^{T} .
\end{aligned}
$$


The parameterization of hierarchical freeform surfaces is characterized by its first fundamental form

$$
\begin{aligned}
& d \widetilde{s}^{2}=\left(\begin{array}{ll}
d s & d t
\end{array}\right)\left(\begin{array}{cc}
\widetilde{E} & \widetilde{F} \\
\widetilde{F} & \widetilde{G}
\end{array}\right)\left(\begin{array}{l}
d s \\
d t
\end{array}\right) \\
& =\left(\begin{array}{ll}
d s & d t
\end{array}\right)\left(\begin{array}{c}
\phi_{s} \\
\phi_{t}
\end{array}\right) \mathbf{I}\left(\begin{array}{ll}
\phi_{s}^{T} & \phi_{t}^{T}
\end{array}\right)\left(\begin{array}{l}
d s \\
d t
\end{array}\right) .
\end{aligned}
$$

Then we obtain the first fundamental matrix of the hierarchical freeform surface as follows

$$
\widetilde{\mathbf{I}}=\left(\begin{array}{c}
\phi_{s} \\
\phi_{t}
\end{array}\right) \mathbf{I}\left(\begin{array}{ll}
\phi_{s}^{T} & \phi_{t}^{T}
\end{array}\right)
$$

If the hierarchical freeform surface is conformal, we have $\widetilde{F}=0$ and $\widetilde{E}=\widetilde{G}$ [14], which lead to

$$
\left\{\begin{array}{l}
\phi_{s} \mathbf{I} \phi_{t}^{T}=0 \\
\phi_{s} \mathbf{I} \phi_{s}^{T}=\phi_{t} \mathbf{I} \phi_{t}^{T}
\end{array}\right.
$$

The derivatives of transformation $\phi$ over variables $s$ and $t$ are as follows.

$$
\phi_{s}=\left(\begin{array}{ll}
u_{s} & v_{s}
\end{array}\right) \text { and } \phi_{t}=\left(\begin{array}{ll}
u_{t} & v_{t}
\end{array}\right)
$$

Substituting Equations (4) into Equations (3), we have

$$
\left(\begin{array}{ll}
u_{s} & v_{s}
\end{array}\right)\left(\begin{array}{cc}
E & F \\
F & G
\end{array}\right)\left(\begin{array}{l}
u_{t} \\
v_{t}
\end{array}\right)=0
$$

and

$$
\left(\begin{array}{ll}
u_{s} & v_{s}
\end{array}\right)\left(\begin{array}{cc}
E & F \\
F & G
\end{array}\right)\left(\begin{array}{l}
u_{s} \\
v_{s}
\end{array}\right)=\left(\begin{array}{ll}
u_{t} & v_{t}
\end{array}\right)\left(\begin{array}{cc}
E & F \\
F & G
\end{array}\right)\left(\begin{array}{l}
u_{t} \\
v_{t}
\end{array}\right) .
$$

From Equation (5), we have

$$
u_{t}=-\frac{v_{t}\left(u_{s} F+v_{s} G\right)}{\left(u_{s} E+v_{s} F\right)} .
$$


Substituting Equation (7) into Equation (6), we obtain $v_{t}$ as follows.

$$
v_{t}= \pm \frac{u_{s} E+v_{s} F}{\sqrt{E G-F^{2}}}
$$

We select the positive sign in Equation (8) such that the derivatives $v_{t}$ and $u_{s}$ have the same variation tendency. By collecting the constraints in Equations (7) and (8), we have

$$
\mathbf{C}(s, t)=\phi_{t}^{T}-\left(\begin{array}{c}
-\frac{F}{\sqrt{E G-F^{2}}}-\frac{G}{\sqrt{E G-F^{2}}} \\
\frac{E}{\sqrt{E G-F^{2}}} \frac{F}{\sqrt{E G-F^{2}}}
\end{array}\right) \phi_{s}^{T}=0,
$$

which always holds at a conformal point.

\section{Conformal Freeform Surfaces}

Conformal parameterization of NURBS surfaces has several special properties, which are extremely valuable for visualization, tessellation and other derivative-based applications. First, conformal parameterization is angle-preserving. Intuitively, suppose $\gamma_{1}, \gamma_{2} \subset[0,1] \times[0,1]$ are two arbitrary intersecting curves in the parameter domain, with the intersection point as $p=\gamma_{1} \cap \gamma_{2}$. Then they are mapped to intersection curves on the $3 \mathrm{D}$ surface $\mathbf{X}, \mathbf{X}(p)=\mathbf{X}\left(\gamma_{1}\right) \cap \mathbf{X}\left(\gamma_{2}\right)$. Suppose at the intersection point $p$, the intersection angle between the tangent vectors $d \gamma_{1}, d \gamma_{2}$ is $\theta$. $\mathbf{X}$ is conformal, if and only if the intersection angle between the tangent vectors $d \mathbf{X}\left(\gamma_{1}\right)$ and $d \mathbf{X}\left(\gamma_{2}\right)$ is also $\theta$, therefore it is shape preserving. For each $(u, v)$, the tangent vectors to the two iso-parametric curves are orthogonal and have the same norm. In other words, a conformal map is locally isotropic, i.e. maps an elementary circle of the $(u, v)$ domain to an elementary circle of the surface. Second, a conformal parameterization will lead to more robust and stable computations for derivative based algorithms such as surface sampling, surface intersection, curvature computation, and so on $[3,31]$.

Given a NURBS surface, this section presents an optimization algorithm to improve its conformality using freeform transformations. To measure the deviation of the current parameterization from conformal parameterizations, the following integral function is adopted

$$
J_{c}\left(\mathbf{Q}_{i, j}\right)=\int_{0}^{1} \int_{0}^{1}\left|\phi_{t}-\left(\begin{array}{c}
-\frac{F}{\sqrt{E G-F^{2}}}-\frac{G}{\sqrt{E G-F^{2}}} \\
\frac{E}{\sqrt{E G-F^{2}}} \frac{F}{\sqrt{E G-F^{2}}}
\end{array}\right) \phi_{s}\right|^{2} d s d t
$$


In general, Equation (10) is highly nonlinear, and there is no closed-form solution for the freeform transformations. Thus we first discretize the above conformal energy by composite Simpson's rule and then its discretized form can be solved using the nonlinear least square method.

\subsection{Numerical Method}

The terms in Equation (10) are approximated by a discrete version of the energy using the composite Simpson's rule [38]. To apply the composite Simpson's rule, we uniformly sample the parameter domain by partitioning the $u$ and $v$ interval into $2 l$ and $2 k$ subintervals

$$
\mathbf{R}_{i, j}=\left(\begin{array}{c}
u_{p}+\frac{i \cdot\left(u_{n_{u}+1}-u_{p}\right)}{2 l} \\
v_{q}+\frac{j \cdot\left(v_{n_{v}+1}-v_{q}\right)}{2 k}
\end{array}\right), \quad i=0, \ldots, 2 l, j=0, \ldots, 2 k
$$

These subdivisions determine the step sizes $h=1 /(2 l)$ and $p=1 /(2 k)$. Let

$$
T=\left|\phi_{t}-\left(\begin{array}{cc}
-\frac{F}{\sqrt{E G-F^{2}}} & -\frac{G}{\sqrt{E G-F^{2}}} \\
\frac{E}{\sqrt{E G-F^{2}}} & \frac{F}{\sqrt{E G-F^{2}}}
\end{array}\right) \phi_{s}\right|^{2} .
$$

The resulting approximation has the form

$$
J=\widetilde{J}+E_{\text {error }}
$$

where

$$
\left\{\begin{aligned}
\widetilde{J}= & \frac{4 h p}{9}\left\{J_{1}+2 J_{2}+4 J_{3}+J_{4}\right\} \\
E_{\text {error }}=-\frac{h^{4} \frac{\partial^{4} T(\bar{s}, \bar{t})}{\partial s^{4}}+p^{4} \frac{\partial^{4} T(\widehat{s}, \vec{t})}{\partial t^{4}}}{180} & \\
J_{1}= & T\left(s_{0,0}, t_{0,0}\right)+2 \sum_{i=1}^{l-1} T\left(s_{2 i, 0}, t_{2 i, 0}\right)+4 \sum_{i=1}^{l} T\left(s_{2 i-1,0}, t_{2 i-1,0}\right)+T\left(s_{2 l, 0}, t_{2 l, 0}\right) \\
J_{2}= & \sum_{j=1}^{k-1} T\left(s_{0,2 j}, t_{0,2 j}\right)+2 \sum_{j=1}^{k-1} \sum_{i=1}^{l-1} T\left(s_{2 i, 2 j}, t_{2 i, 2 j}\right)+ \\
& 4 \sum_{j=1}^{k-1} \sum_{i=1}^{l} T\left(s_{2 i-1,2 j}, t_{2 i-1,2 j}\right)+\sum_{j=1}^{k-1} T\left(s_{2 l, 2 j}, t_{2 l, 2 j}\right) \\
J_{3}= & \sum_{j=1}^{k} T\left(s_{0,2 j-1}, t_{0,2 j-1}\right)+2 \sum_{j=1}^{k} \sum_{i=1}^{l-1} T\left(s_{2 i, 2 j-1}, t_{2 i, 2 j-1}\right)+ \\
& 4 \sum_{j=1}^{k} \sum_{i=1}^{l} T\left(s_{2 i-1,2 j-1}, t_{2 i-1,2 j-1}\right)+\sum_{j=1}^{k} T\left(s_{2 l, 2 j-1}, t_{2 l, 2 j-1}\right) \\
J_{4}= & T\left(s_{0,2 k}, t_{0,2 k}\right)+2 \sum_{i=1}^{l-1} T\left(s_{2 i, 2 k}, t_{2 i, 2 k}\right)+4 \sum_{i=1}^{l} T\left(s_{2 i-1,2 k}, t_{2 i-1,2 k}\right)+T\left(s_{2 l, 2 k}, t_{2 l, 2 k}\right)
\end{aligned}\right.
$$


for some $(\bar{s}, \bar{t})$ and $(\widehat{s}, \hat{t})$ in the $(\mathrm{s}, \mathrm{t})$ parameter domain. We can increase the sampling density $l$ and $k$ to decrease the error term $E_{\text {error }}$. When using $l, k=$ 10 , the error term has the following form

$$
E_{\text {error }}=-\frac{\frac{\partial^{4} \mathbf{T}(\bar{u}, \bar{v})}{\partial u^{4}}+\frac{\partial^{4} \mathbf{T}(\widehat{u}, \widehat{v})}{\partial v^{4}}}{2.88 \times 10^{11}} .
$$

For a given NURBS surface, the numerator of $E_{\text {error }}$ is bounded and $E_{\text {error }}$ is seven order of magnitude smaller than that bound with the sampling density $l, k=10$. The energy function in Equation (10) is then discretized as

$$
\widetilde{J}_{c}\left(\mathbf{Q}_{i, j}\right) \quad i=0 \ldots n_{s}, j=0 \ldots n_{t} .
$$

Minimizing the Energies in Equation (12) leads to the following optimization problem

$$
\min _{\mathbf{Q}_{i, j}} \widetilde{J}_{c}
$$

which is a nonlinear least square problem. This target function can be minimized numerically using the Levenberg-Marquardt method, which enjoys robust global convergence property to a local minimum. Each iteration of the Levenberg-Marquardt method needs to evaluate the gradient of function $\widetilde{J}_{c}$ with respect to the control points $\mathbf{Q}_{i, j}$ of the freeform transformation. For numerical optimization, it is advisable to start from an initial point which is not far away from a local minimum. We determine the initial transformation by the following least square method. First, we uniformly sample a grid in the $(u, v)$ parameter domain of the NURBS surface and obtain the 2D conformal mapping $\mathbf{M}$ of their corresponding 3D discretized mesh on the NURBS surface by the Ricci flow method [15]. After the 2D conformal mapping is obtained, the initial freeform transformation can then be constructed by approximating the composite mapping $\mathbf{M} \circ \mathbf{X}$ using the least square method. After the initial freeform transformation is obtained, we optimize the nonlinear least square problem in Equation (13) using the Levenberg-Marquardt method. The Levenberg-Marquardt algorithm is a popular method for nonlinear least square optimization. It is more robust than the Gauss-Newton algorithm, and is guaranteed to converge to a local minimum [22]. The Levenberg-Marquardt method interpolates between the Gauss-Newton algorithm and the method of gradient descent. The Levenberg-Marquardt method is more robust than the Gauss-Newton algorithm, which means that in many cases it finds a solution even if it starts very far off the final minimum. Levenberg-Marquardt method can also be viewed as Gauss-Newton using a trust region approach. There is no guarantee that it will converge to a global minimum. To find the global minima, we need to use global optimization algorithms, which require a global search approach and are usually time-consuming [20]. Instead, our method selects a good initial point for the Levenberg-Marquardt algorithm, so that it 
can converge to a local minimum nearby. Such strategy has been successfully applied to other geometric modeling problems involving nonlinear least square optimization $[8,24,54,55]$. For a specific freeform transformation, the conformality deviation of the resultant hierarchical freeform surface will not be equal to zero even after the nonlinear optimization procedure. To obtain a hierarchical freeform surface with bounded conformality deviations, the nonlinear least square conformality minimization and the freeform transformation refinement interleave during the optimization procedure, which is described in detail in the next subsection.

\subsection{Approximation within a specified accuracy}

In practice, we want to optimize the transformation of given freeform surfaces such that the hierarchical freeform representation converges to be conformal. The conformality of the hierarchical freeform surface can be checked by uniformly sampling points inside the $(s, t)$ parameter domain and computing the maximal conformal deviation at the sampling points

$$
\max _{0 \leq k \leq m}\left|\mathbf{N} \times \frac{\partial \mathbf{X}}{\partial s}-\frac{\partial \mathbf{X}}{\partial t}\right|^{2},
$$

where $\mathbf{N}$ is the unit normal vector of the freeform surface at the current point and $\frac{\partial \mathbf{X}}{\partial s}$ and $\frac{\partial \mathbf{X}}{\partial t}$ are the two partial derivatives of the surface over variables $s$ and $t$, respectively. If the maximal conformal deviation is larger than the given tolerance $\epsilon_{c}$, perform the following iterative steps.

1. Refine the freeform transformation.

2. Compute the freeform transformation by the nonlinear least square optimization method.

3. Check the conformal deviation of the new hierarchical freeform surface.

4. If the maximal conformal error is less than the tolerance $\epsilon_{c}$, exit; otherwise, goto Step 1.

By performing the above refinement and optimization procedures iteratively, we obtain a conformal hierarchical freeform surface with bounded conformal deviations at the end.

\section{Experimental results}

Our algorithm is implemented on a PC with Intel Duo CPU 3.06 GHZ, 2G Memory and Microsoft Visual Studio 2008. To show the performance of our 

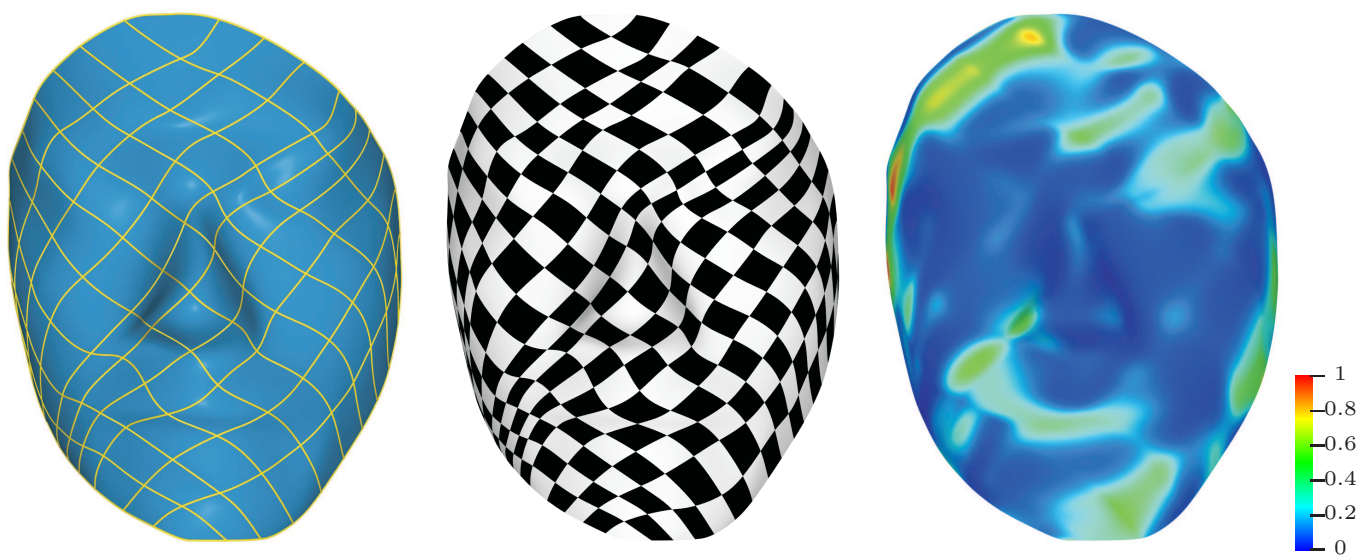

(a) original surface parameterization
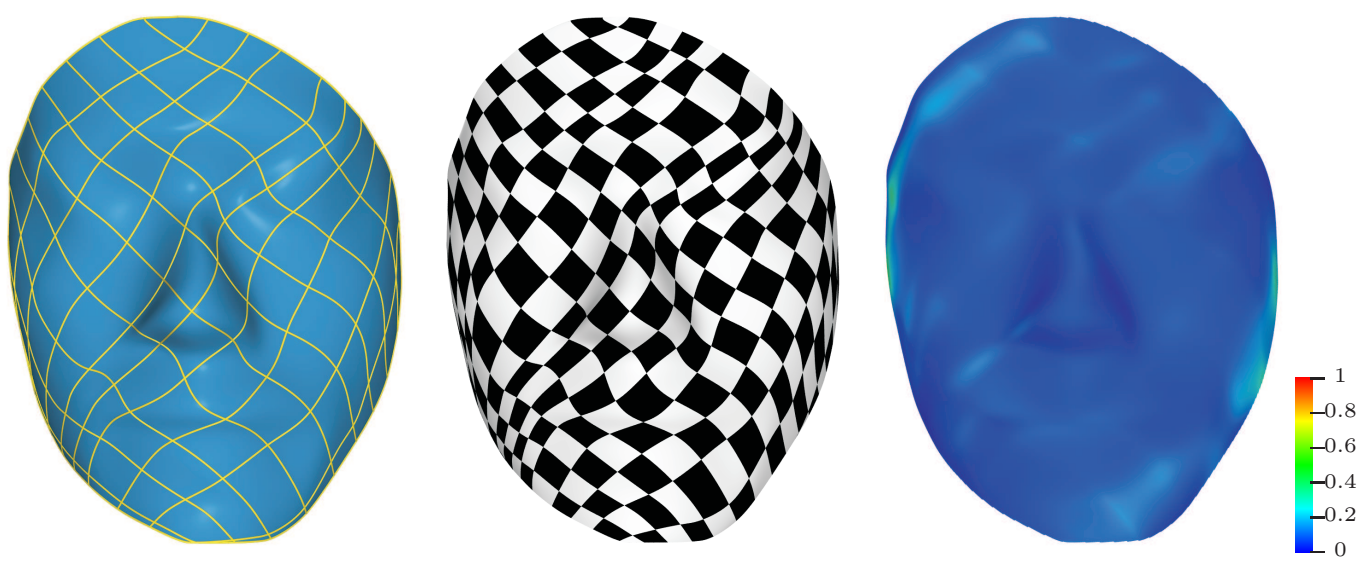

(b) surface parameterization optimized by general bilinear transformation
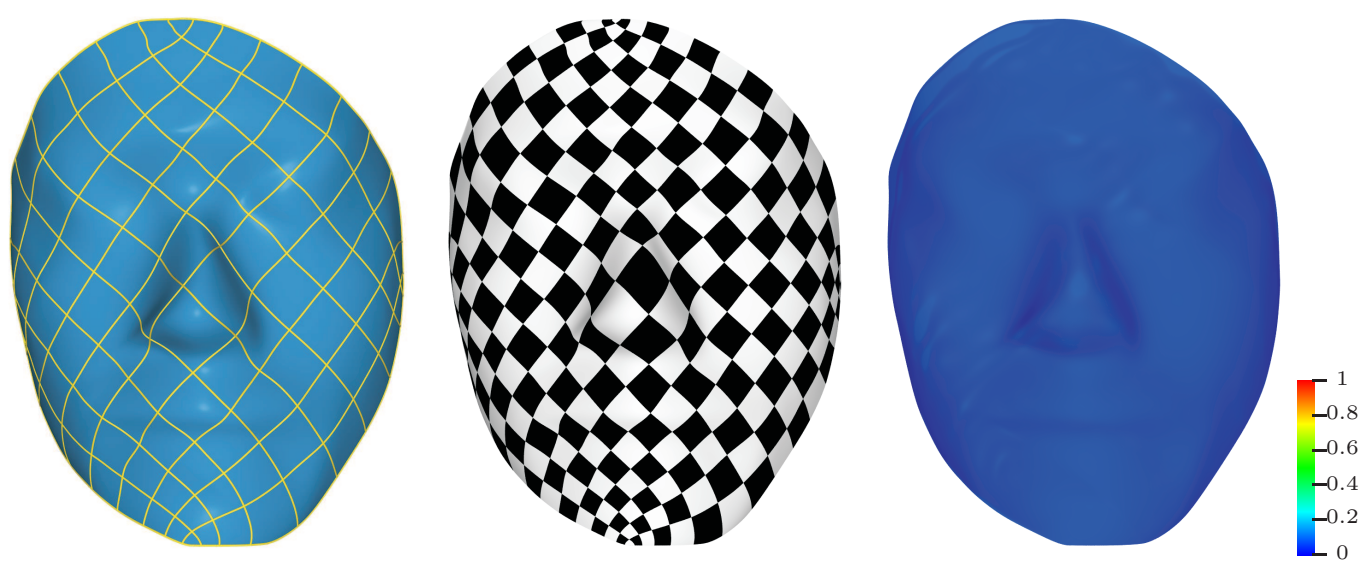

(c) surface parameterization optimized by freeform transformation

Fig. 3. Optimization of a face surface with 43 by 43 control points. (a) the isoparametric curves, the texture mapping and the color-coded conformality of the original parameterization; (b) the iso-parametric curves, the texture mapping and the color-coded conformality of the parameterization optimized by the general bilinear transformation; (c) the iso-parametric curves, the texture mapping and the color-coded conformality of the parameterization optimized by the freeform transformation. 

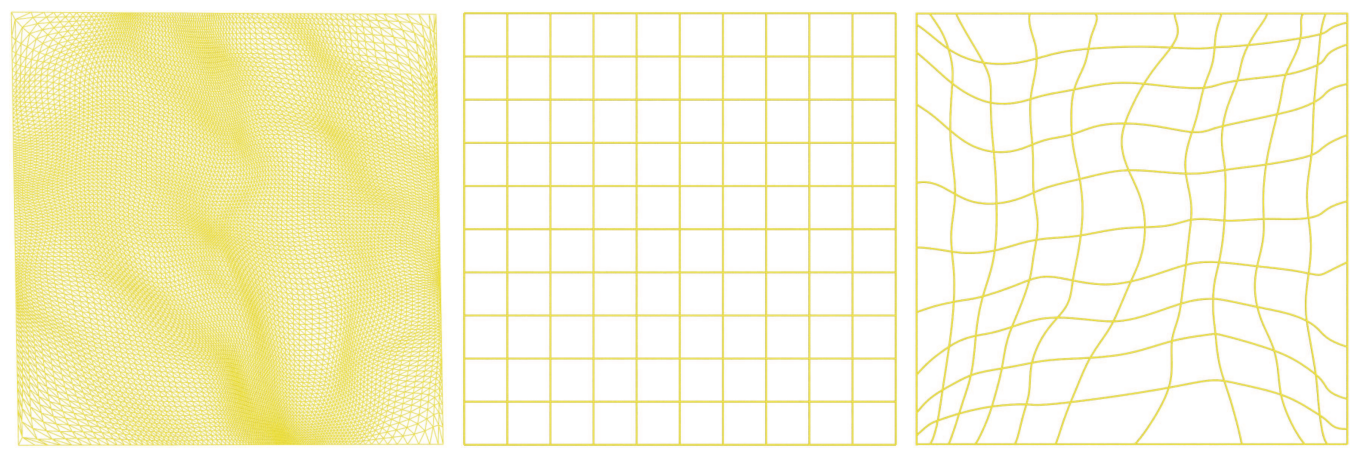

Fig. 4. The freeform transformation of the face NURBS surface. The conformal mapping of the 3D discrete mesh, the iso-parametric lines in the parameter domain of the final freeform transformation and the iso-parametric curves of the freeform transformation are arranged from left to right.
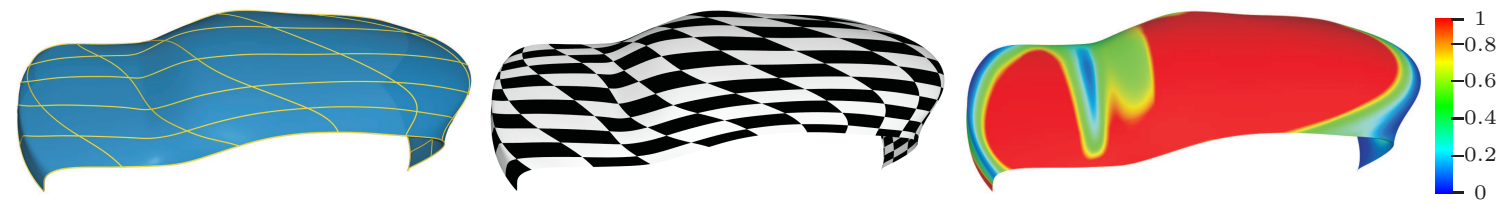

(a) original surface parameterization
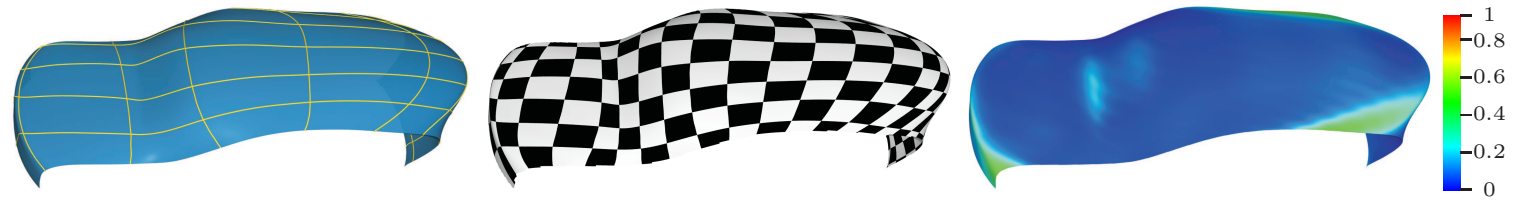

(b) surface parameterization optimized by general bilinear transformation
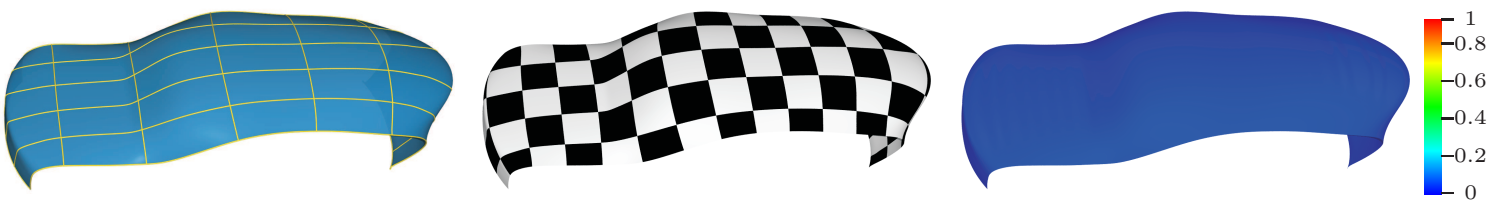

(c) surface parameterization optimized by freeform transformation

Fig. 5. Optimization of a car top surface with 43 by 43 control points. (a) the iso-parametric curves, the texture mapping and the color-coded conformality of the original parameterization; (b) the iso-parametric curves, the texture mapping and the color-coded conformality of the parameterization optimized by the general bilinear transformation; (c) the iso-parametric curves, the texture mapping and the color-coded conformality of the parameterization optimized by the freeform transformation.

algorithm, several examples are given as follows. Here we adopt the sampling density $l, k=10$ (see Section 3.1) for all the examples.

Figure 3 illustrates the optimization result of a face NURBS surface with 43 by 43 control points. To show the performance, each surface is illustrated using three methods: iso-parametric curves, texture mapping and color-coded conformality. The iso-parametric curve network can also be seen as quad tes- 

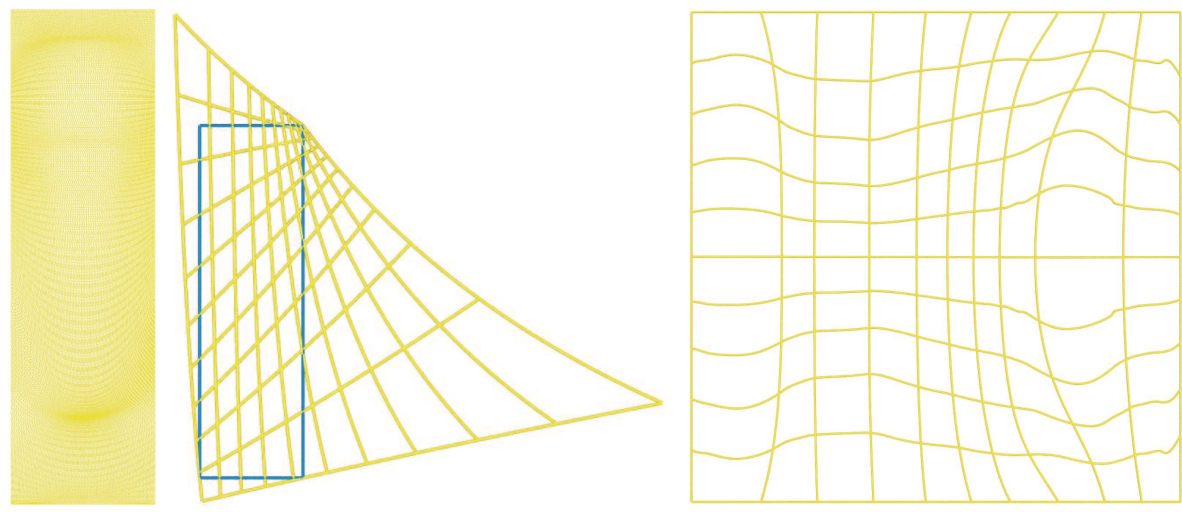

Fig. 6. The transformation of the car top NURBS surface. The conformal mapping of the $3 \mathrm{D}$ discrete mesh, the iso-parametric lines of the general bilinear transformation (the blue rectangle is the boundaries of the original parameter domain), and the iso-parametric curves of the freeform transformation are arranged from left to right.

sellations of the given freeform surfaces. Let $N(\delta, \zeta)$ denote the unit normal to the surface. In the color-coded images, we measure for a parameterization $\mathbf{X}(\delta, \zeta)$ the following conformality function across the surface

$$
E(\delta, \zeta)=\left\|N(\delta, \zeta) \times \frac{\partial X}{\partial \delta}(\delta, \zeta)-\frac{\partial X}{\partial \zeta}(\delta, \zeta)\right\|,
$$

and use colors to illustrate the value of $E(\delta, \zeta)$ (blue is good conformality and red is bad conformality). The surface parameterization $\mathbf{X}(\delta, \zeta)$ is conformal at a point $(\delta, \zeta)$ if and only if $E(\delta, \zeta)=0$.

As shown in Figure 3, the face NURBS surface is optimized using the general bilinear transformation and the freeform transformation, respectively. From Figure 3, it can be seen that the original surface parameterization is far from being conformal. Texture mapping of the original parameterization involves a lot of shape distortion and color-coded image of the original parameterization includes a couple of green areas. By the Ricci flow method in [15], we compute the conformal mapping of its discretized mesh (see Figure 4). By approximating the conformal mapping using the least square method, we obtain the initial freeform transformation with maximal conformality deviation 0.16 , which is further optimized and refined until the conformal deviation is smaller than the given user tolerance $\epsilon_{c}=0.05$. From the iso-parametric curves, the texture mapping and the color-coded conformality in Figure 3, we can see that the conformality of the surface can be improved to some extent by the general bilinear transformation. To better illustrate the conformality deviation, the maximal conformality deviation of the original surface is normalized to 1 for all examples of this section. Though the maximal conformality deviation of the resultant surface optimized by the general bilinear transformation is reduced to 0.41 , the texture mapping result still involves a lot of shape distortion and the color-coded image has several green areas near the boundary. 

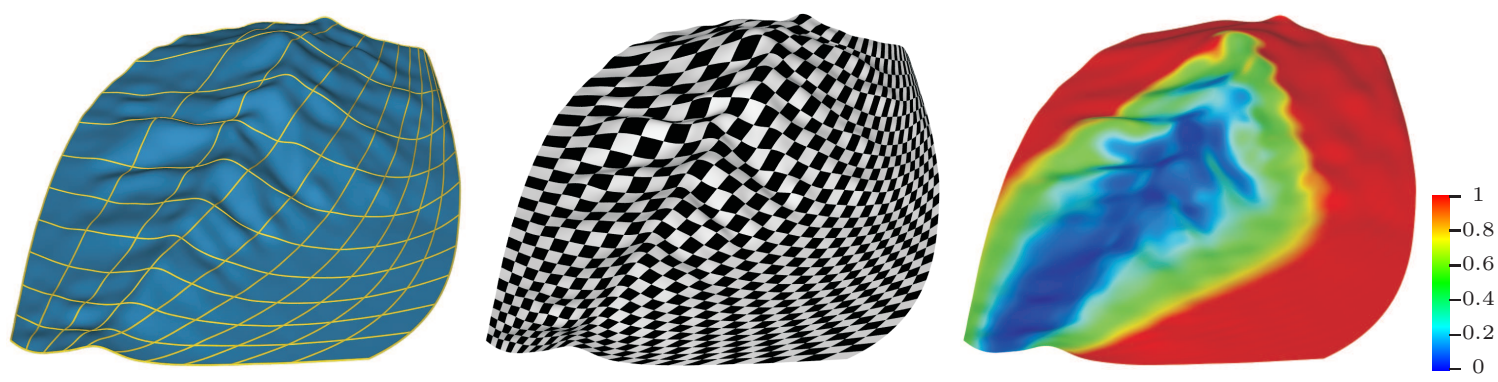

(a) original surface parameterization
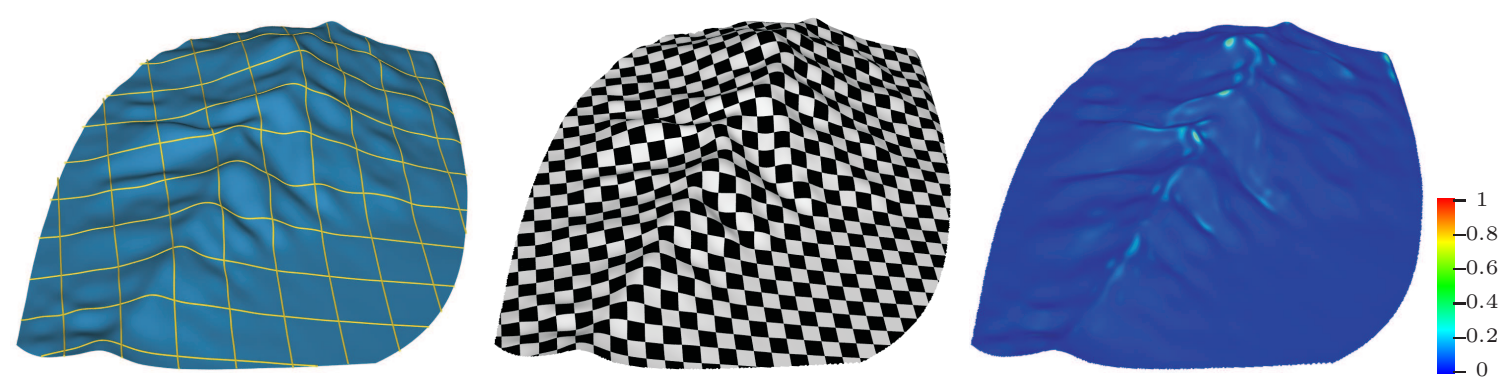

(b) surface parameterization optimized by general bilinear transformation
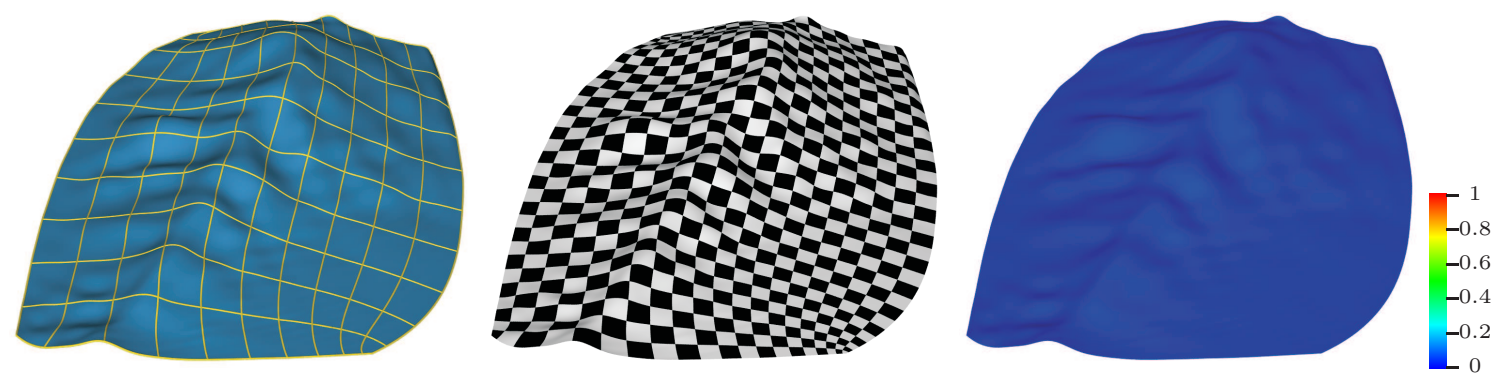

(c) surface parameterization optimized by freeform transformation

Fig. 7. Optimization of a terrain surface with 34 by 34 control points. (a) the iso-parametric curves, the texture mapping and the color-coded conformality of the original parameterization; (b) the iso-parametric curves, the texture mapping and the color-coded conformality of the parameterization optimized by the general bilinear transformation; (c) the iso-parametric curves, the texture mapping and the color-coded conformality of the parameterization optimized by the freeform transformation.

Compared with the general bilinear transformation, the freeform transformation is much more powerful. By setting the conformality tolerance $\epsilon_{c}=0.05$, we obtain the optimal freeform transformation with 17 by 17 control points. After the parameterization optimization by the freeform transformation, the texture mapping of the optimized parameterization well keeps the local shape and the color-coded conformality image of the optimized parameterization is almost blue.

Another example is given in Figure 5, where a car top surface with 34 by 34 control points is optimized using the general bilinear transformation and the 


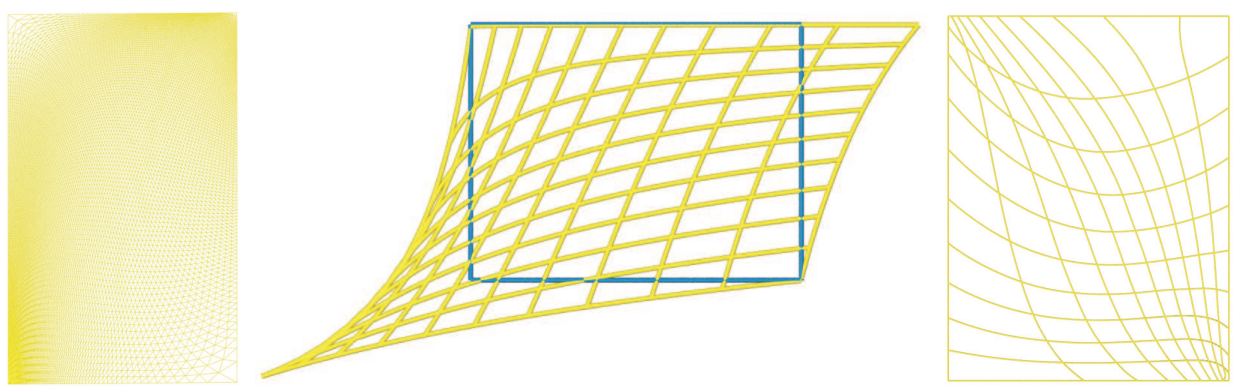

Fig. 8. The freeform transformation of the terrain NURBS surface. The conformal mapping of the 3D discrete mesh, the iso-parametric lines of the general bilinear transformation (the blue rectangle is the boundaries of the original parameter domain) and the iso-parametric curves of the freeform transformation are arranged from left to right.

freeform transformation. The conformal mapping of its 3D discretized mesh, the final general bilinear transformation and the freeform transformation are illustrated in Figure 6. From the results in Figure 5, we can see that both the general bilinear transformation and the freeform transformation can improve the conformality of the given car top surface. By using the general bilinear transformation, the conformality deviation of the given car top surface is reduced to 0.17 . However, the texture mapping result and the color-coded conformality image still involve large shape distortion and green areas near the boundaries of the tail, respectively. Compared with the limited ability of the general bilinear transformation, the maximal conformality deviation of the car top surface has been reduced to 0.09 by the initial freeform transformation, which is further optimized to the user specified tolerance $\epsilon_{c}=0.02$ by the final freeform transformation with 32 by 32 control points. Both the texture mapping and the color-coded conformality images demonstrate the effectiveness of the freeform transformation.

Both the above face and car top examples have regular surface boundaries (almost a rectangle). To show the ability of our method to handle NURBS surfaces with irregular boundaries, a terrain NURBS surface is given in Figure 7 , in which we measure the data near the interesting mountain area and reconstruct the NURBS surface from its triangular mesh using a least square method in [35]. From Figure 7, partially due to its irregular boundaries, the results of rendering and tessellation applications are far from being satisfied. Both the general bilinear transformation and freeform transformation methods are applied to optimize the conformality of the terrain surface. Though the conformality of the terrain surface is largely improved by the general bilinear transformation, the resultant color-coded image still has green areas along the mountain ridge. The freeform transformation exhibits to be more powerful and flexible than the general bilinear transformation. Especially, the freeform transformation improves the surface conformality while keeping the surface boundaries. Also to show the efficiency and effectiveness of our method 


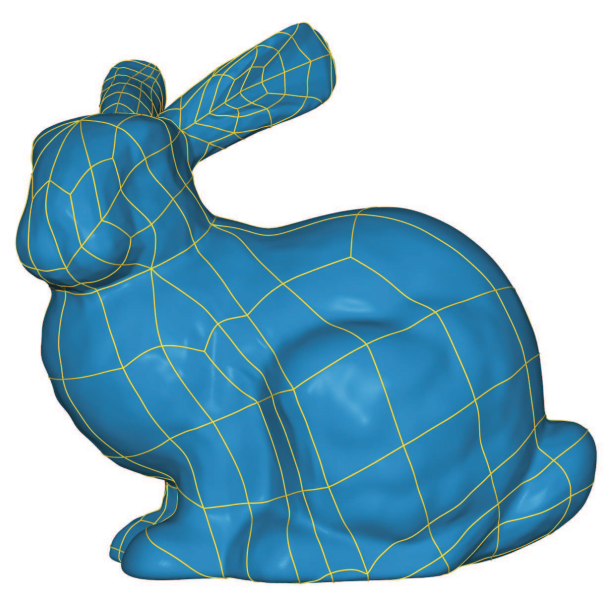

(a)

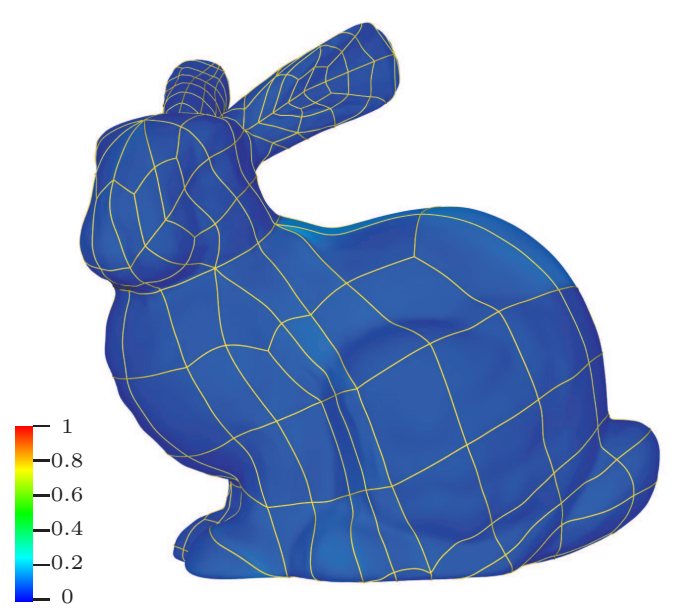

(c)

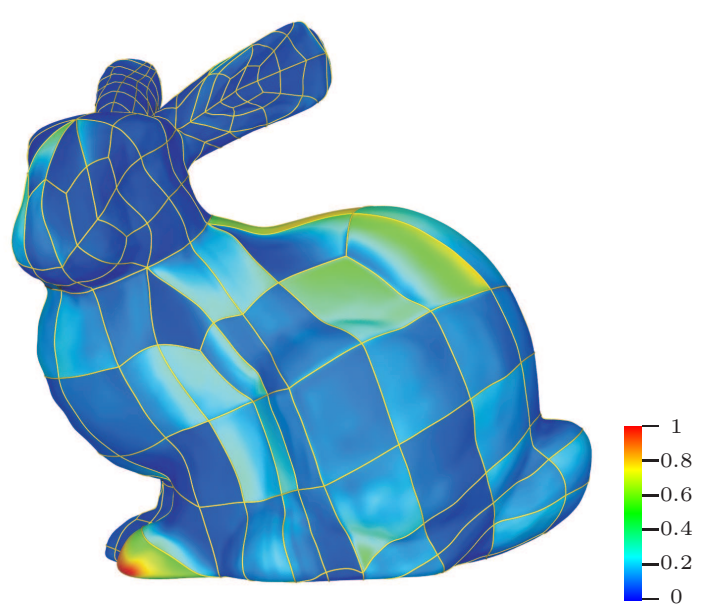

(b)

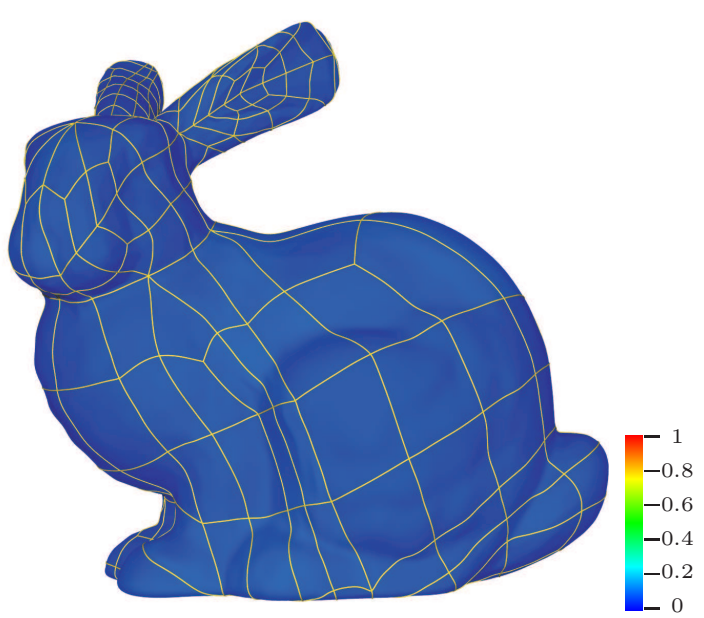

(d)

Fig. 9. The transformation of the bunny model: (a) the bunny model (the yellow curves are the subsurface boundaries); (b) the conformality of the original bunny model; (c) the conformality of the resultant model optimized by the general bilinear transformations; (d) the conformality of the resultant model optimized by the freeform transformations.

to improve the conformality of complex and multiple freeform surfaces, an example of the bunny model with 316 patches is given in Figure 9. The layout of the subsurface is illustrated in Figure 9(a) and the conformality of the bunny model is improved by the general bilinear transformation and the freeform transformation. For the complex model with multiple patches, the parametric continuity between adjacent surfaces is combined with the conformality energy during the optimization procedure. The color-coded conformality images are illustrated in Figure 9, from which it can be seen that the freeform transformation is superior to the general bilinear transformation at improving the surface conformality near the bunny neck.

The above four examples show that the freeform transformation can reduce 


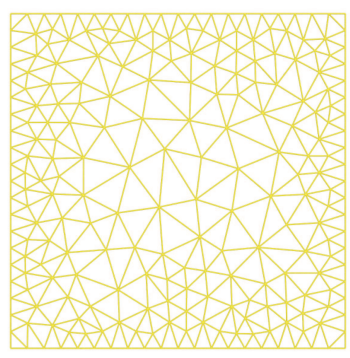

(a)

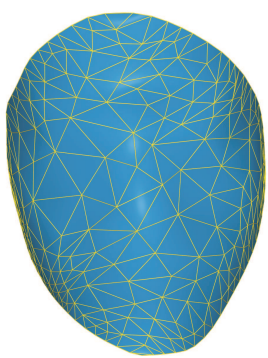

(b)

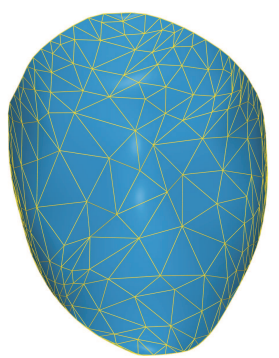

(c)

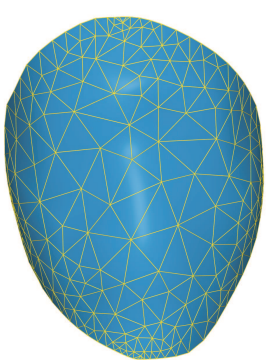

(d)

Fig. 10. Face triangulation.(a)2D triangulation with minimal angle $33.9^{\circ}$; (b) the surface tessellation by mapping the $2 \mathrm{D}$ triangulation in (a) on the original surface with minimal angle $2.2^{\circ}$; (c) the surface tessellation by mapping the $2 \mathrm{D}$ triangulation in (a) on the optimized surface using the general bilinear transformation with minimal angle $4.1^{\circ}$; (d)the surface tessellation by mapping the $2 \mathrm{D}$ triangulation in (a) on the hierarchical freeform surface with minimal angle $20.3^{\circ}$.
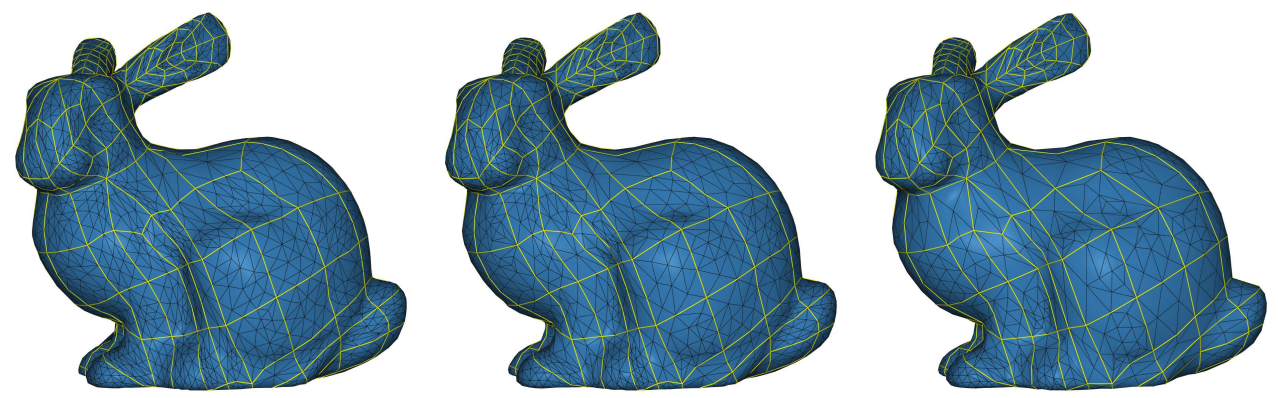

Fig. 11. Tessellation of the bunny model. Left: the original model; middle: the optimized surface by general bilinear transformation; right: the hierarchical freeform surface.
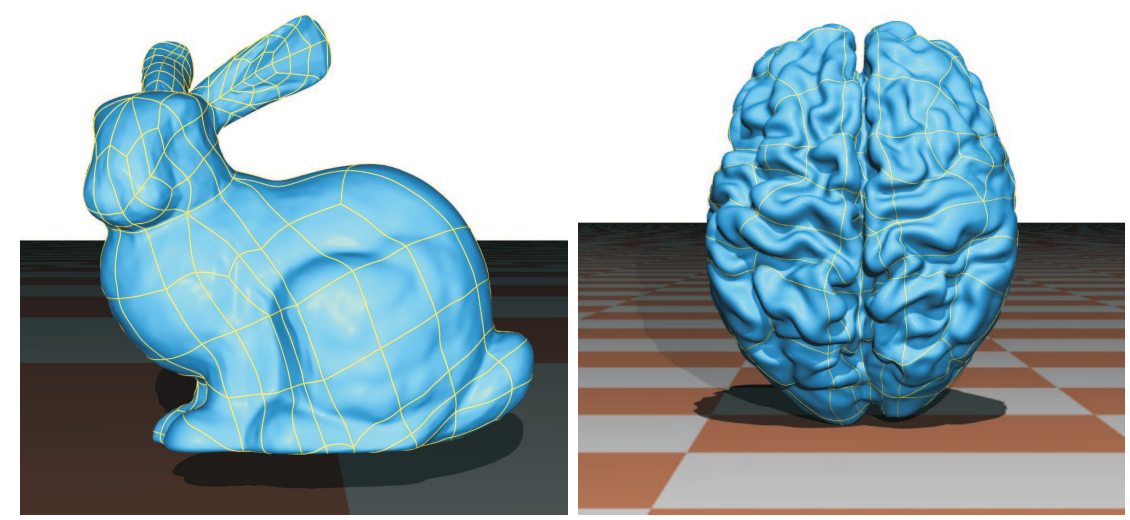

Fig. 12. Ray tracing of surfaces.

the conformality deviation of given freeform surfaces to the user specified tolerance. Compared with the general bilinear transformation, the freeform transformation can improve the surface conformality while keeping its boundaries. Also, the time cost of bilinear transformation and the freeform transformation 
Table 1

Time cost (ms) of reparameterization methods

\begin{tabular}{lcccc}
\hline & face & cartop & terrain & bunny \\
\hline general bilinear transformation & 31 & 40 & 36 & 350 \\
freeform transformation & 65 & 87 & 76 & 455 \\
\hline
\end{tabular}

Table 2

Tessellation results of the bunny model with edge and face tolerances 0.001 .

\begin{tabular}{lccc}
\hline & original model & general bilinear tran. & hierarchical freeform model \\
\hline vertex number & 3089 & 2389 & 1637 \\
face number & 6172 & 4774 & 3270 \\
mesh deviation & 0.00095 & 0.00096 & 0.00092 \\
minimal angle & $3.9^{\circ}$ & $5.3^{\circ}$ & $11.6^{\circ}$ \\
time (ms) & 35 & 43 & 47 \\
\hline
\end{tabular}

for the above four examples are given in Table 1. From Table 1, we can see that the parameterization quality of the resultant surface is much better than the previous results in [55] while the time cost of the freeform transformation is comparable to that of the general bilinear transformation.

To show the efficiency of the hierarchical freeform surface for tessellation application, an example is given to tessellate the face surface in Figure 3. The parameter domain is first triangulated using the constrained Delaunay triangulation algorithm in [40] by setting the minimal angle $\theta=33.9^{\circ}$. Then the $2 \mathrm{D}$ triangles are mapped to the original surface, the optimized surface by general bilinear transformation and the hierarchical freeform surface (see Figure 10). From Figure 10, the hierarchical freeform surface preserves the triangle angle better than the original surface and the optimized surface using the general bilinear transformation. We can see that the quality of the triangulation highly depends on the surface parameterization. Furthermore, we demonstrate the superiority of the hierarchical freeform surface to handle more complex trimmed NURBS surfaces. For trimmed NURBS surfaces, the tessellation method presented in [2] is implemented on the original surface, the optimized surface by the general bilinear transformation and the hierarchical freeform surface, where the geometric approximation errors for the stitching boundaries and the surfaces are controlled under the given tolerances (see Figure 11). The main advantage of the method in [2] is that it produces much fewer triangles than traditional methods [46], which is preferred for subsequent CAD applications. The trimming curves are first approximated by polylines under a prescribed distance tolerance, which is followed by the NURBS surface tessellation based on kd-tree subdivisions. The key step of the surface tessellation in [2] is to generate the constrained triangulation of the sampling point set 
of the trimming curves and surfaces. As the constrained triangulation of point clouds is a non-trivial problem, practically all NURBS tessellation algorithms generate the final triangulation in the parameter domain of the surface, which is reasonable as long as the surface does not deform the polygon too much. As the parameter domain of the hierarchical freeform surface better keeps the shape of the NURBS surface geometry (more conformal parameterization), less triangles will be introduced to tessellated the NURBS models under the specified tolerances, which is verified by the examples given in Figure 11. The computation time, the number of triangles, the minimal angle and the mesh deviation are reported in Table 2. From Table 2, we can see that the hierarchical freeform surface will introduce less triangles than the original surface and the optimized surface by general bilinear transformation under the same subdivision tolerances, which demonstrates the superiority of the hierarchical freeform surface in surface tessellation application.

In computer graphics, ray tracing is a technique for generating an image by tracing the path of light through pixels in an image plane and simulating the effects of its encounters with virtual objects. The technique is capable of producing a very high degree of visual realism, usually higher than that of typical scanline rendering methods, but at a greater computational cost. The intersection between the ray tracing lines and the model accounts for most of the time cost. To show the efficiency of the hierarchical freeform surface for rendering, the bunny model in Figure 9 and a brain model are rendered using the ray tracing method in [1] (see Figure 12), where the NURBS surfaces are ray traced by intersection algorithms between rays and NURBS surfaces without the preprocess of tessellating the NURBS surfaces. Directly rendering free-form surfaces has several advantages compared with traditional methods, which convert the NURBS surfaces into triangles before rendering. The timeconsuming triangulation preprocessing can be omitted and the accuracy is not limited to a certain distance from the observer. For the direct rendering of NURBS surfaces in [1], roughly $60 \%$ of the CPU time is spend in the surface evaluation, including the calculation of partial derivatives. Therein, a Newton iteration-based intersection approach is used for the intersection test between the rays and NURBS surfaces. The parameterization quality highly affects the convergence speed of the intersection algorithms and surfaces with better parameterization are advantageous for intersection computation due to smoother intersection curves and faster algorithm convergence, which has been verified and reported in [7]. For the original surface, the optimized surface by the general bilinear transformation and the hierarchical freeform surface, the time cost of the rendering algorithm in [1] is illustrated in Table 3 . The rendering image resolution is set to $1600 \times 1200$. The bunny model is composed of 316 NURBS patches while the brain model has 202 NURBS pathes. From Table 3, we can see that the superiority of hierarchical freeform surfaces to the general bilinear method is obvious for the rendering application. 
Table 3

Rendering time cost (s) of ray tracing method

\begin{tabular}{lcc}
\hline & bunny & brain \\
\hline original surface & 833 & 554 \\
optimized surface by general bilinear transformation & 415 & 308 \\
hierarchical freeform surface & 45 & 36 \\
\hline
\end{tabular}

Besides the conformality, equiareality should also be considered in the freeform surface construction. In most cases, the freeform surfaces are not developable. Thus we cannot obtain both conformal and area preserving parameterizations. The conformality of the freeform surfaces is important for applications such as rendering (texture mapping), tessellation (keeping angles and shapes of the triangle), surface intersections and so on. Also the equiareality is important for sampling/tessellation applications (sampling density). There is a tradeoff between the conformality and equiareality of the freeform surfaces. In this paper, we focus on the conformality of the freeform surfaces. To improve the equiareality of the freeform surfaces is left as a future work. Also feature awareness of the surface parameterization is another important issue, which should be considered for the spline construction.

\section{Conclusions}

In order to improve conformality of given NURBS surfaces, an optimization algorithm is presented in this paper based on freeform transformations (hierarchical freeform surfaces). A nonlinear energy measuring the conformality deviations and its numerical approximation are formulated. To minimize the discretized version of the conformal energy, the initial freeform transformation is first obtained by approximating the conformal mapping of the 3D discretized mesh, which is computed using Ricci flow method. To further improve the conformality of the initial freeform transformation, the nonlinear conformality optimization and the transformation refinement interleave until the conformality deviation is reduced to the user specified tolerance. Several examples are given to show the performance of our algorithm for rendering and tessellation applications.

\section{Acknowledgements*}

This work was supported by NSF CCF-1544267, the China National Natural Science Foundation (61202146, 61272243, 61472224, and 61472225), Shandong 
Key Research and Development Program (2015GGX106006), the special funding of independent innovation and transformation of achievements in Shandong Province under Grant No. 2014ZZCX08201, the National High-Tech Research and Development Plan of China under Grant No.2014AA01A302, the special funds of Taishan scholar construction project, Young Scholars Program of Shandong University under Grant No.2015WLJH41.

\section{References}

[1] Abert O, Geimer M and Muller S. Direct and Fast Ray Tracing of NURBS. IEEE Symposium on Surfaces Interactive Ray Tracing 2006:161-168.

[2] Balzs A, Guthe M and Klein R. Efficient trimmed NURBS tessellation. Journal of WSCG 2004; 12(1):27-33.

[3] Cheung G, Lau R and Li F. Incremental rendering of deforming NURBS surfaces. In VRST 03: Proceedings of the ACM symposium on Virtual reality software and technology (New York, NY, USA, 2003), ACM Press, pp. 48-55.

[4] Chuang JH, Lin $\mathrm{CH}$ and Hwang WC. Variable-radius blending of parametric surfaces. The Visual Computer 1995;11(10):513-525.

[5] Chuang JH and Hwang WC. Variable-radius blending by constrained spine generation. The Visual Computer 1997;13(7):316-329.

[6] Costantini P, Farouki RT, Manni C and Sestini A. Computation of optimal composite re-parameterizations. Computer Aided Geometric Design 2001;18(9):875-897.

[7] Casciola G and Morigi S. Reparameterization of NURBS curves. International Journal of Shape Modeling 1996;2(2-3):103-116.

[8] Deng B, Pottmann H, Wallner J. Functional webs for freeform architecture. Computer Graphics Forum 2011;30(5):1369-1378.

[9] Do Carmo MP. Differential Geometry of Curves and Surfaces, Prentice Hall, 1976.

[10] Farin G. NURBS from Projective Geometry to Practical Use, second ed., A K Peters, 1999.

[11] Farin G. Rational quadratic circles are parametrized by chord length. Computer Aided Geometric Design 2006; 23(9):722-724.

[12] Farouki RT and Sakkalis T. Real rational curves are not 'unit speed', Computer Aided Geometric Design 1991;8(2):151-157.

[13] Farouki RT. Optimal parameterizations. Computer Aided Geometric Design 1997;14(2):153-168. 
[14] Floater MS and Hormann K. Surface parameterization: a tutorial and survey. In advances in multiresolution for geometric modelling, Dodgson NA, Floater MS, and Sabin MA, Eds., Mathematics and Visualization. Springer, Berlin, Heidelberg, 2005, 157-186.

[15] Gu DX, Zeng W, Luo F, and Yau ST. Numerical computation of surface conformal mappings. Computational Methods and Functional Theory (CMFT) $2011 ; 11(2): 747 \mathrm{C} 787$.

[16] Hamann B and Tsai PY. A tessellation algorithm for the representation of trimmed nurbs surfaces with arbitrary trimming curves. Computer-Aided Design 1996;28(6/7):461-472.

[17] He L, Schaefer S and Hormann K. Parameterizing subdivision surfaces. ACM Transactions on Graphics (TOG) 2010;29(4).

[18] He L, Loop C and Schaefer S. Improving the parameterization of approximate subdivision surfaces. Computer Graphics Forum 2012;31(7):2127-2134.

[19] Hormann K, Lévy B and Sheffer A. Mesh parameterization: Theory and practice. In SIGGRAPH 2007 Course Notes 2007;2:1-122.

[20] Horst R, Pardalos PM and Thoai NV. Introduction to Global Optimization, Second Edition, Springer, 2000.

[21] Jüttler B. A vegetarian approach to optimal parameterizations. Computer Aided Geometric Design 1997;14(9):887-890.

[22] Kanzow C, Yamashita N and Fukushima M. Levenberg-marquardt methods with strong local convergence properties for solving nonlinear equations with convex constraints. Journal of Computational and Applied Mathematics 2004;173(2):321-343.

[23] Kumar S and Manocha D. Efficient rendering of trimmed NURBS surfaces. Computer-Aided Design 1995;27(7):509-521.

[24] Liu Y, Pottmann H, Wallner J, Yang YL and Wang W. Geometric modeling with conical meshes and developable surfaces. ACM Transactions on Graphics 2006;25(3):681-689.

[25] Lee ETY and Lucian ML. Möbius reparametrizations of rational B-splines. Computer Aided Geometric Design 1991;8(3):213-215.

[26] Lévy B, Petitjean S, Ray N and Maillot J. Least Squares Conformal Maps for Automatic Texture Atlas Generation. ACM Transactions on Graphics 2002, 21(3):362-371. Proceedings of SIGGRAPH 2002.

[27] Lourakis M. levmar: Levenberg-Marquardt nonlinear least squares algorithms in $\mathrm{C} / \mathrm{C}++$. http://www.ics.forth.gr/ lourakis/levmar/.

[28] Lu W. Curves with chord length parameterization. Computer Aided Geometric Design 2009;26(3):342-350. 
[29] Michael E, Martin K, Alexander S, Niloy JM, Helmut P and Mark P. Paneling architectural freeform surfaces ACM Transactions on Graphics 2010;29(4).

[30] Mullen P, Tong YY and Alliez P. and Desbrun, M. Spectral Conformal Parameterization. Computer Graphics Forum 2008;27(5):1487-1494.

[31] Ng WMM and Tan ST. Incremental tessellation of trimmed parametric surfaces. Computer-Aided Design 2000;32(4):279-294.

[32] Nocedal J and Wright SJ. Numerical Optimization, second ed., Springer,2006.

[33] Ong BH. An extraction of almost arc-length parameterization from parametric curves. Ann. Numer. Math. 1996;3:305-316.

[34] Piegl LA and Richard AM. Tessellating trimmed NURBS surfaces. ComputerAided Design 1995;27(1):15-26.

[35] Piegl LA and Tiller W. The NURBS Book, second ed., Springer, New York, 1997.

[36] Piegl LA and Tiller W. Filling $n$-sided regions with NURBS patches. The Visual Computer 1999;15(2):77-89.

[37] Ray N, Li WC, Levy B, Sheffer A and Alliez P. Periodic global parameterization. ACM Transactions on Graphics 2006;25(4):1460-1485.

[38] Richard LB and John DF. Numerical analysis, Cengage Learning, 2010.

[39] Sheffer A, Praun E and Rose K. Mesh parameterization methods and their applications. Foundations and Trends in Computer Graphics and Vision 2006;2(2):105-171.

[40] Shewchuk JR. Delaunay Refinement Algorithms for Triangular Mesh Generation. Computational Geometry: Theory and Applications 2002;22(13):21-74.

[41] Shpitalni M, Koren Y and Lo CC. Realtime curve interpolators. ComputerAided Design 1994;26(11):832-838.

[42] Trefethen LN and Bau D. Numerical linear algebra. SIAM, 1997.

[43] Wang FC, Yang DCH. Nearly arc-length parameterized quintic spline interpolation for precision machining. Computer-Aided Design 1993;25(5):281288.

[44] Wang FC, Wright PK, Barsky BA and Yang DCH. Approximately arclength parameterized $C^{3}$ quintic interpolatory splines. ASME J. Mech. Design 1999;121:430-439.

[45] Wever U. Optimal parameterization for cubic spline. Computer-Aided Design 1991;23(9):641-644.

[46] William LL. Tessellation of trimmed NURB surfaces. Computer Aided Geometric Design 1996;13(2):163-177. 
[47] Yang DCH and Kong T. Parametric interpolator versus linear interpolator for precision CNC machining. Computer-Aided Design 1994;26(3):225-234.

[48] Yang DCH and Wang FC. A quintic spline interpolator for motion command generation of computer-controlled machines. ASME J. Mech. Design 1994;116:226-231.

[49] Yang YJ, Yong JH, Zhang H, Paul JC and Sun JG. A rational extension of Piegl's method for filling n-sided holes. Computer-Aided Design 2006;38(11):1166-1178.

[50] Yang YJ, Yong JH, Zhang H, Paul JC and Sun JG. Optimal parameterizations of Bézier surfaces. In: 2nd International Symposium on Visual Computing (ISVC06); 2006.p. 672-681.

[51] Yang YJ, Zhang H, Yong JH, Paul JC and Sun JG. Constrained Delaunay triangulation using Delaunay visibility. In: 2nd International Symposium on Visual Computing (ISVC06); 2006.p. 682-691.

[52] Yang YJ, Zeng W, Zhang H, Paul JC and Yong JH. Projection of Curves on B-Spline Surfaces Using Quadratic Reparameterization. Journal of Graphical Models 2010;72(5):47-59.

[53] Yang YJ, Zeng W, Yang CL, Meng XX, Yong JH and Deng BL. G1 continuous approximate curves on NURBS surfaces. Computer-Aided Design 2012;44(9):824-834.

[54] Yang YJ, Zeng W, Yang CL, Deng BL, Meng XX and lyengar SS. An algorithm to improve parameterizations of rational Bezier surfaces using rational bilinear reparameterization. Computer-Aided Design 2013;45(3):628-638.

[55] Yang YJ, Zeng W and Song TQ. Optimizing conformality of NURBS surfaces by general bilinear transformations. Computer-Aided Design 2015;46(6):12-25.

[56] Yeh SS and Hsu PL. The speed-controlled interpolator for machining parametric curves. Computer-Aided Design 1999;31(5):349-357.

[57] Zou G, Hu JX, Gu XF and Hua J. Authalic parameterization of general surfaces using Lie advection. IEEE Trans. Vis. Comput. Graph 2011;17(12):2005-2014. 